\title{
EDITORIAL
}

\author{
Dra. Patricia Gallego Torres \\ Grupo ENCIMA - Editora \\ Doctorado Interinstitucional en Educación DIE - UD
}

\begin{abstract}
En esta edición especial, la revista Investigación Científica, de la Universidad Distrital Francisco José de Caldas presenta, algunas perspectivas en la enseñanza de las ciencias, las tecnologías y las matemáticas. Este número nació con la finalidad de servir como cauce para acercar, y en lo posible conectar, diversas disciplinas científicas que se trabajan al interior de algunos grupos de investigación de la Facultad de Ciencias y Educación de la Universidad Distrital, así como para divulgar y debatir algunos temas de investigadores colaboradores que interactúan con estos grupos.
\end{abstract}

El Consejo editorial de la revista y el Centro de Investigaciones y Desarrollo Científico, esperemos que en el futuro muchos otros grupos no solo de la Facultad de Ciencias y de Educación sino de las otras facultades, se animen a editar otro número y que sea la ocasión para que se convierta en una serie que muestre el estado de la investigación de la Universidad Distrital Francisco José de Caldas.

Con este número especial que presentamos y que responden en algunos casos a los problemas de investigación de las tesis doctorales de los énfasis de ciencias y matemáticas del Doctorado Interinstitucional en Educación sede Universidad Distrital Francisco José de Caldas, que forma parte de la nueva generación de investigaciones, como se verá a través del mismo, lo que se busca en definitiva, es establecer un dialogo abierto con la comunidad académica y proponer elementos de reflexión y análisis de los temas que aquí se abordan.

Por último queremos agradecer, primer lugar, a los autores que desde sus investigaciones, quisieron aportar al número enviando artículos del estado del arte de sus trabajos. En segundo lugar, quisiera agradecer el nivel de entusiasmo y apoyo recibido de los evaluadores, y para terminar, al CIDC por la oportunidad de hacer realidad este número especial sobre la enseñanza de las ciencias, las matemáticas y las tecnologías. 


\section{INSTRUCTIVO PARA AUTORES}

Recepción de artículos: Los artículos presentados para su publicación deben ser ensayos, o resultados de investigaciones, inéditos o no publicados en castellano sobre investigaciones relacionadas con las ciencias, las matemáticas las tecnologías y la educación científica, los cuáles serán sometidos a un proceso de doble arbitraje ciego que evaluará la originalidad del texto, su desarrollo, la calidad de su argumentación y su relevancia. Los autores cuyos artículos publiquemos ceden los derechos de los artículos publicados a la revista y al Centro de Investigaciones y Desarrollo Científico de la Universidad Distrital Francisco José de Caldas.

Envío de artículos: Los artículos deben ser remitidos por correo electrónico a: adpgallegot@ udistrital.edu.co con copia a: centroi@udistrital.edu.co.

Arbitraje: Todos los artículos recibidos, que cumplan con los requisitos formales de presentación, son sometidos aun sistema de doble evaluación ciega de alguno de los integrantes del comité de evaluadores. En casos dudosos será sometido a una tercera evaluación.
Bibliografía: Es necesaria y se ubica al final de cada artículo. Va ordenada alfabéticamente por el apellido del autor, y empleamos el sistema APA.

Extensión: Los artículos tendrán una extensión máxima de 20 páginas incluyendo la bibliografía a espacio 1.5 y letra Times Román en formato Word.

Títulos: Sugerimos que los títulos no excedan de 15 palabras. Este debe incluir su traducción al Ingles.

Resúmenes: No deben tener más de 150 palabras y debe incluir de 5 a 7 palabras claves, y su correspondiente traducción al inglés.

Gráficos y Tablas: Las tablas, gráficos, fotografías y dibujos además de incluirlas en el artículo, deberán ser enviadas en un archivo adjunto.

Recomendaciones: Citar la revista así: Revista Científica, N. , páginas

Enviar la hoja de vida de sintetizada (máximo 2 páginas) de los autores.

\section{EVAALUADORES ARTICCULOS \\ REVISTA CIENTIEICA, $\mathbb{N}^{\circ} 11$}

Dra. Adriana Patricia Gallego Torres DIE-UD

Magíster Sergio Briceño

UnIVERSIDAd Distrital.

Magíster Ruth Molina V. UnIIERSIDAD DISTRITAL.

Magíster Pedro Rocha Salamanca UnIVERSIDAd DISTRITAL.

Magíster Duván Reyes R. Universidad Distrital.

Magíster Fernando Guerrero UnIVERSIDAD DISTRITAL.
Dr. Bruno Demore UNIVERSIDAD de Bologna- ITALIA

Magíster Royman Pérez Universidad Pedagógica Nacional

Magíster Rómulo Gallego Badillo Universidad Pedagógica Nacional

Magíster Germán Londoño UNIVERSidAd de Valencia- EsPaña

Magíster Roberto Figueroa Universidad del AtLántico
Magíster Mónica Rueda Pinto Universidad Santo Tomás.

Dr. Alejandro Vásquez Pontifica Universidad Javeriana.

Magíster Catalina Lizcano UNIVERSIDAd de La SABana.

Magíster Diana Sepúlveda UNIVERSidAd de La SABANa

Magíster Adriana Valenzuela UnIVERSidAd de LA SABANA 